\title{
Calidad en Colangiografía endoscópica
}

\author{
Aurelio López-Colombo* \\ Departamento de Gastroenterología, Hospital Ángeles Puebla, Puebla, México
}

La Organización Mundial de la Salud (OMS) señala que para otorgar servicios médicos de calidad se requiere que la atención sea segura, efectiva, oportuna, eficiente, equitativa y centrada en el paciente. La seguridad se refiere a minimizar los riesgos y daños a los usuarios. La efectividad se refiere a que la atención se base en el conocimiento científico y las guías de práctica clínica. La oportunidad se refiere a reducir retrasos en la atención. La eficiencia se refiere a la optimización de los recursos y a evitar desperdicios. La equidad se refiere a que los servicios se otorguen con la misma calidad independientemente del género, raza, grupo étnico, ubicación geográfica y nivel socioeconómico. Atención centrada en el paciente se refiere a que se toman en cuenta las preferencias y aspiraciones individuales de los pacientes y se respete su autonomía. Considerando que la colangiografía endoscópica (CPRE) es uno de los procedimientos endoscópicos técnicamente más demandantes y de más alto riesgo, las asociaciones de endoscopia más importantes como la Sociedad Americana de Endoscopía Gastrointestinal (ASGE) y la Sociedad Europea de Endoscopía Gastrointestinal (ESGE), tomando en cuenta las directrices de la OMS han propuesto una serie de indicadores de calidad o medidas de desempeño para asegurar que este procedimiento sea realizado con la mayor efectividad y seguridad para el paciente ${ }^{1,2}$. Estas recomendaciones sin duda son una referencia para todos los endoscopistas que realizan este procedimiento y son la base para este resumen en el que se seleccionaron algunos trabajos presentados en la Semana
Gastroenterológica de la Unión Europea 2019 (UEGW) y la Semana de Enfermedades Digestivas de los EEUU 2020 (DDW).

Durante la UEGW, un grupo de investigadores presentó un análisis de las tendencias de demandas por responsabilidad profesional médica en gastroenterología presentadas en los EE.UU. ${ }^{3}$. Se trató de un estudio retrospectivo que comparó las características de las demandas en gastroenterología en dos periodos de tiempo 2006-2010 y 2011-2015. De un total de 90,743 demandas por responsabilidad médica, el $2.07 \%$ correspondió a gastroenterología. De ellas las que pagaron una indemnización promedio más alta fueron las demandas por procedimientos de las vías biliares, particularmente CPRE y la comparación entre los periodos mostró que la tendencia va en aumento. Independientemente de que la cultura de la demanda no se aplica de igual forma a muchos países, este trabajo nos muestra que la CPRE es uno de los procedimientos donde con frecuencia no se obtiene la satisfacción del paciente. Los autores sugieren que además tener el entrenamiento adecuado, se debe tener particular cuidado en seleccionar la indicación apropiada para el procedimiento. Esto último es precisamente uno de los indicadores de calidad pre-procedimiento considerado prioritario por la ASGE: la frecuencia con la que la CPRE se realiza por una indicación publicada en guías como una indicación apropiada debe ser superior al $90 \%{ }^{1}$. Una de las indicaciones más frecuentes para la realización de CPRE es coledocolitiasis. Sin embargo, no es infrecuente que pacientes con sospecha de coledocolitiasis sean sometidos a CPRE y no 
solo no se encuentren litos, sino que además presenten complicaciones. En el 2010, la ASGE publicó recomendaciones para realización de CPRE en sospecha de coledocolitiasis, pero recientemente publicó una actualización ${ }^{4,5}$. Durante la DDW se presentaron 4 trabajos que abordaron este tema. Rashtak $\mathrm{S}$, et al. en un análisis retrospectivo que evaluó 639 pacientes de primera vez que fueron sometidos a CPRE por sospecha de coledocolitiasis en 14 hospitales, encontraron que el $43 \%$ de los casos no se apegaron a las recomendaciones de la ASGE 2010. La falta de adherencia a estas guías fue significativamente más alta en centros comunitarios que en hospitales académicos ${ }^{6}$. Los otros tres trabajos tuvieron como objetivo evaluar el desempeño y exactitud de las recomendaciones de la ASGE 2019 al compararlas con las recomendaciones $2010^{7-9}$. Los 3 trabajos coincidieron en que los criterios 2019 son menos sensibles pero más específicos que los criterios 2010, por lo que su aplicación minimizaría la realización de CPRE innecesarias. El estudio de Aamar A., et al. mostró que la utilización de los criterios de alto riesgo 2019 aumento la especificidad para el diagnóstico de coledocolitiasis de $56 \%$ a $82 \%$ con respecto a los criterios $2010^{7}$. Los resultados fueron semejantes en el estudio de Hasak, et al., en el que el aumento en la especificidad fue de 55.2 a $74.4 \%{ }^{8}$. El estudio de Chandran, et al. identificó que con los criterios de alto riesgo ASGE 2010 el 76.5\% de los pacientes sometidos a CPRE por sospecha de coledocolitiasis verdaderamente tuvo litos, mientras que con los criterios ASGE 2019 la proporción aumentó a $82.1 \%{ }^{9}$.

La seguridad, sin duda, es una de las preocupaciones más importantes en este procedimiento, por lo que otro de los indicadores de calidad considerados prioritarios, tanto para la ASGE como para la ESGE, es la tasa de pancreatitis post CPRE por tratarse de la complicación más frecuente. Para la ESGE, la tasa ideal de pancreatitis post CPRE debería ser menor al $5 \%$, aunque acepta como medida de desempeño una tasa menor al $10 \%$. Existe evidencia que respalda ciertas medidas de prevención de pancreatitis que están publicados en guías de la ASGE y la ESGE ${ }^{10,11}$. Varios trabajos se presentaron en la UEGW y la DDW que abordaron este tema. Dos trabajos, que utilizaron la misma metodología (meta análisis en red), concluyeron que la combinación de hidratación intravenosa e indometacina rectal fue el método más eficaz para prevenir pancreatitis. El primero trabajo, de un grupo húngaro, fue presentado en la UEGW y evaluó 6 combinaciones de tratamientos: Ringer lactato solo (RS), solución salina sola (SS), indometacina rectal sola (IR), no tratamiento (NT), $R L+I R$ y SS + IR. Se evaluaron 16 ensayos clínicos que incluyeron un total de 6070 CPRE. El análisis mostró que la combinación $R L+I R$ fue significativamente más eficaz para reducir el riesgo de pancreatitis que el resto de las terapias (OR: 0.06; IC 95\%: 0.00-0.69) ${ }^{12}$. El segundo estudio fue realizado por un grupo americano y presentado en la DDW. Este también fue un meta análisis en red en el que se analizaron 48 ensayos clínicos que evaluaron las siguientes intervenciones; antiinflamatorios, prótesis pancreática, hidratación venosa o combinaciones de estas en un total de 13,808 pacientes. La tasa de pancreatitis en el grupo placebo fue de $12.3 \%$. De acuerdo con una escala de desempeño, la combinación de SS + IR tuvo la mayor probabilidad de ser considerada la mejor intervención profiláctica; superior a antiinflamatorios solos, prótesis pancreática u otras combinaciones ${ }^{13}$.

Con lo que respecta a aspectos técnicos, no era posible pasar por alto un título tan provocador como el del siguiente resumen: Do we really want to prevent post ERCP pancreatitis- why not to start with needle knife precut? Se trató de un ensayo clínico aleatorizado realizado en la India y presentado en la DDW, en el que se comparó una de las recomendaciones de la ESGE: realización de precorte temprano (posterior a dos intentos de canulación) (grupo A, 152 pacientes) contra la maniobra experimental: precorte con cuchillo de manera inicial (grupo B, 151 pacientes). La tasa de éxito de canulación del conducto biliar fue de $61.3 \%$ en el grupo A vs. $98.6 \%$ en el grupo B ( $p=0.001$ ), lo cual incrementó a $98 \%$ en el grupo A después de la realización de precorte. La tasa de pancreatitis fue de $5.2 \%$ vs. $0.8 \%(p=0.04)$ en el grupo $A$ y $B$ respectivamente. Los autores concluyen que el precorte con cuchillo de manera inicial se asocia a un menor riesgo de pancreatitis ${ }^{14}$. Sin embargo, estos resultados contrastan con los de otro estudio presentado también en la DDW con características semejantes. Se trató de un ensayo clínico aleatorizado realizado por un grupo de Pensilvania que comparó fistulotomía con cuchillo vs. acceso transpapilar. Este grupo no encontró diferencias ni en la tasa de éxito para acceder a la vía biliar ni en la tasa de complicaciones, incluyendo pancreatitis. Vale la pena señalar que, en este estudio, el número de individuos por grupo fue notablemente menor que del estudio previo (38 vs. 40 ) por lo que posiblemente no se alcanzó el poder para demostrar diferencias. ${ }^{15}$ Por lo tanto la pregunta a contestar en el futuro próximo será: ¿deberíamos realizar precorte de manera inicial en todos las CPRE en las que el objetivo es acceder a la vía biliar? 


\section{Conflicto de intereses}

Los autores declaran no tener conflicto de intereses alguno.

\section{Financiamiento}

\section{Los autores no recibieron patrocinio para llevar a cabo este artículo.}

\section{Bibliografía}

1. Adler DG, Lieb JG 2nd, Cohen J, et al. Quality indicators for ERCP. Gastrointest Endosc 2015;81(1):54-66.

2. Domagk D, Oppong KW, Aabakken L, et al. Performance measures for ERCP and endoscopic ultrasound: a European Society of Gastrointestinal Endoscopy (ESGE) Quality Improvement Initiative. Endoscopy 2018; 50(11): 1116-1127.

3. Jayaraj M, Ohning GV, Parikh D, et al. Recent Trends in Gastroenterology and Endoscopy related Medical Professional Liability claims in the United States of America. United European Gastroenterol J. 2019;7(8 suppl):401.

4. ASGE Standards of Practice Committee; Maple JT, Ben-Menachem T, Anderson MA, et al. The role of endoscopy in the evaluation of suspected choledocholithiasis. Gastrointest Endosc 2010;71(1):1-9.

5. ASGE Standards of Practice Committee, Buxbaum JL, Abbas Fehmi SM, Fishman DS, et al. ASGE Guideline on the Role of Endoscopy in the Evaluation and Management of Choledocholithiasis. Gastrointest Endosc 2019;89(6):1075-1105
6. Rashtak S, Chandran A, Dedania B et al. Practice patterns and adherence to society guidelines for suspected choledocholithiasis in a large health care system of 14 hospitals; comparing academic and community settings. Gastrointest Endosc 2020;91(5s): AB530.

7. Aamar A, Barakat M, Aloreidi K, et al. Performance of updated ASGE high-risk criteria for choledocholithiasis. Gastrointest Endosc 2020;91(5s): AB375.

8. Hasak S, Busebee B, Sloan I, et al. Validation of the predictors of choledocholithiasis and proposed management algorithm from the 2019 ASGE guideline "Role of endoscopy in the evaluation and management of choledocholithaisis». Gastrointest Endosc 2020;91(5s): AB358-359.

9. Chandran A, Rashtak S, Dedania B, et al Comparison of performance and accuracy of revised 2019 ASGE guidelines in predicting choledocholithiasis against 2010 ASGE guidelines: outcomes from a large hospital system comprising 14 hospitals. Gastrointest Endosc 2020;91(5s): AB506.

10. ASGE Standards of Practice Committee, Chandrasekhara V, Khashab MA, Muthusamy VR, et al. Adverse events associated with ERCP. Gastrointest Endosc. 2017;85(1):32-47.

11. Dumonceau JM, Andriulli A, Elmunzer BJ, et al. Prophylaxis of post-ERCP Pancreatitis: European Society of Gastrointestinal Endoscopy (ESGE) Guideline - Updated June 2014. Endoscopy 2014;46(9):799-815.

12. Márta K, Gede N Szakács Z et al. Combination of intravenous hydration and indomethacin suppository is the most effective therapy for the prevention of post-ercp pancreatitis- a network meta-analysis. United European Gastroenterol J. 2019;7(8_suppl):226.

13. Akshintala VS, Sperna Weiland CJ, Bhullar FA, et al. The combination of intravenous fluid and indomethacin is more efficacious than pancreatic stents and NSAIDs alone for the prevention of post-ercp pancreatitis: a network meta-analysis of randomized controlled trials. Gastroenterology 2020;158(6):S1135-1136.

14. Maharshi S, Sharma SS. Do we really want to prevent post ERCP pancreatitiswhy not to start with needle knife precut? Gastrointest Endosc 2020;91(5s): AB102.

15. Bakhit M, Mitsuhashi S, Novikov AA, et al. Is needle-knife fistulotomy (NKF) a safe and effective approach for primary biliary access? A randomized controlled trial comparing NKF to standard cannulation performed by NKF naive advanced endoscopist. Gastrointest Endosc 2020:91(5s):AB371. 